EPJ Web of Conferences 112,01001 (2016)

DOI: 10.1051/epjconf/201611201001

(C) Owned by the authors, published by EDP Sciences, 2016

\title{
Resonance matrix elements on the lattice
}

\author{
Andria Agadjanov ${ }^{1, a}$, Véronique Bernard ${ }^{2, b}$, Ulf-G. Meißner ${ }^{1,3, c}$, and Akaki Rusetsky ${ }^{1, d}$ \\ ${ }^{1}$ Helmholtz-Institut für Strahlen- und Kernphysik (Theorie), Bethe Center for Theoretical Physics, Universität \\ Bonn, D-53115 Bonn, Germany \\ ${ }^{2}$ Institut de Physique Nucléaire, UMR 8608, CNRS, Univ. Paris-Sud, Université Paris-Saclay, F-91406 Orsay \\ Cedex, France \\ ${ }^{3}$ Institute for Advanced Simulation (IAS-4), Institut für Kernphysik (IKP-3) and Jülich Center for Hadron \\ Physics, Forschungszentrum Jülich, D-52425 Jülich, Germany
}

\begin{abstract}
The notion of resonance matrix elements is discussed. The analogue of the Lellouch-Lüscher formula for the pion photo(electro)production amplitude in the $\Delta(1232)$ resonance region is given. A prescription for the pole extraction of the $\Delta N \gamma^{*}$ transition form factors is provided. Theoretical prospects on the lattice calculation of $B \rightarrow K^{*}(892) l^{+} l^{-}$form factors are briefly summarized.
\end{abstract}

\section{Introduction}

Recently, the $\Delta N \gamma^{*}$ transition form factors have been calculated on the lattice [1,2]. The knowledge on these form factors, in particular, is important to calculate the quark transition charge density, which could be visualized [3]. However, due to large values of the quark masses in these simulations, the $\Delta$ is treated as a stable particle. Consequently, the standard well-known techniques of lattice QCD are applicable. This does not hold anymore for such values of the pion mass, where the $\Delta$ resonance eventually decays.

The resolution of the problem is given by the Lellouch-Lüscher method [4], which relates the electroweak decay amplitude to the finite-volume matrix element of the weak Hamiltonian. The method properly takes into account the different normalizations of the matrix elements in the infinite and finite volume.

Moreover, a meaningful definition should be given to the matrix elements involving resonances, such as the $\Delta$ or $\rho$. It is well known that resonances manifest themselves as complex poles of the scattering T-matrix on unphysical Riemann sheets. It appears natural to introduce the resonance matrix elements in a similar manner [5,6]. Such a definition involves an analytic continuation of the matrix element to the resonance pole position. Thus, the corresponding form factor could be only defined at the resonance pole. Other possible definitions would be model- or process-dependent.

In the present write-up, we review the results of Ref. [6] on the photo(electro)production $\gamma^{*} N \rightarrow$ $\pi N$ near the $\Delta(1232)$ resonance. We apply systematically a non-relativistic effective field theory in a

\footnotetext{
ae-mail: aagadjanov@hiskp.uni-bonn.de

be-mail: bernard@ipno.in2p3.fr

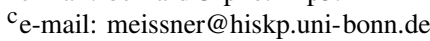

de-mail: rusetsky@hiskp.uni-bonn.de
} 
finite volume in the covariant version [12,13]. It is shown, along the lines of the Lellouch-Lüscher method, how the corresponding multipole amplitudes can be found from the lattice data. We discuss also the extraction of the $\Delta N \gamma^{*}$ transition form factors at the resonance pole. At the end, we give a brief outlook.

\section{The $\Delta N \gamma^{*}$ transition on the lattice}

\subsection{Infinite volume}

The $\Delta N \gamma^{*}$ transition is governed by the three-point function of the form

$$
F^{\mu \rho}(P, Q)=i^{2} \int d^{4} X d^{4} Y e^{i P X-i Q Y}\left\langle 0\left|T O^{\mu}(X) J^{\rho}(0) \bar{\psi}(Y)\right| 0\right\rangle,
$$

where $O^{\mu}(X)$ and $\psi(Y)$ denote the $\Delta$ and nucleon local field operators, respectively, and $J^{\rho}$ is the electromagnetic current. By separating the contribution to this three-point function near a double pole, we can define the current matrix element involving the resonance as

$$
\left\langle P, \lambda\left|J^{\rho}(0)\right| Q, \varepsilon\right\rangle=\lim _{P^{2} \rightarrow s_{R}, Q^{2} \rightarrow m_{N}^{2}} \frac{Z_{R}^{-1 / 2}}{2 E_{R}} \frac{Z_{N}^{-1 / 2}}{2 m_{N}}\left(s_{R}-P^{2}\right)\left(m_{N}^{2}-Q^{2}\right) \bar{u}_{\mu}(P, \lambda) F^{\mu \rho}(P, Q) u(Q, \varepsilon) .
$$

Here, $Z_{R}$ and $Z_{N}$ denote the renormalization constants of the $\Delta$ and nucleon fields, respectively, $s_{R}$ is the complex $\Delta$ resonance pole, and $m_{N}$ is the nucleon mass. $u_{\mu}(P, \lambda)$ describes the free spin-3/2 particle with complex mass $E_{R}$ and spin projections $\lambda=-3 / 2,-1 / 2,1 / 2,3 / 2$. Similarly, $u(Q, \varepsilon)$ is a free Dirac spinor with $\varepsilon=-1 / 2,1 / 2$. Although the resonance can not be associated with any single-particle state in the Fock space of the theory, nevertheless, Eq. (2) gives a meaning to the corresponding resonance matrix element. To the best of our knowledge, this is the only field-theoretical definition possible. Clearly, the definition Eq. (2) is process-independent.

On the other hand, the presence of the resonance is usually associated with the change of the scattering phase shift by the $\pi / 2$ degree value. Then, in the narrow width approximation, the imaginary part of the amplitude $\mathcal{A}$ can be related to the corresponding current matrix element $F$ (see Refs. $[9,10])$ :

$$
|\operatorname{Im} \mathcal{A}|=\sqrt{\frac{8 \pi}{p_{A} \Gamma}}\left|F^{A}\right|,
$$

where all quantities are taken at the real Breit-Wigner pole $p=p_{A}$ and $\Gamma$ is the resonance width. However, since the non-resonant background is unknown, the extracted form factors will be processdependent.

In principle, both definitions converge for infinitely narrow resonances [6]. In practice, the result of Ref. [13] which extracts the residues of the photoproduction amplitude at the complex pole positions corresponding to the different baryon resonances from the experimental data, suggests that for some resonances the discrepancies in the results could be sizable.

Below, we provide formulae, which can be used on the real axis, as well as in the complex energy plane. Still, we emphasize again, that only the definition, which implies the analytic continuation, leads to the process-independent values of the resonance form factors. 


\subsection{Finite volume}

The Lorentz decomposition of the $\Delta N \gamma^{*}$ current matrix element requires three scalar form factors $G_{M}(t), G_{E}(t), G_{C}(t)$, where $t=Q^{2}$ is a photon four-momenta squared. In order to determine them separately, we choose the following kinematics: we consider the $\Delta$ at rest $\mathbf{P}=0$ and put the 3momenta of the nucleon $\mathbf{Q}$ along the third axis. Then, after rotation to the Euclidean space, we pick up the following matrix elements

$$
\begin{gathered}
\left\langle 1 / 2\left|J^{3}(0)\right| 1 / 2\right\rangle=\frac{E_{\Delta}-Q^{0}}{E_{\Delta}} A G_{C}(t), \\
\left\langle 1 / 2\left|J^{+}(0)\right|-1 / 2\right\rangle=\sqrt{\frac{1}{2}} A\left(G_{M}(t)-3 G_{E}(t)\right), \\
\left\langle 3 / 2\left|J^{+}(0)\right| 1 / 2\right\rangle=\sqrt{\frac{3}{2}} A\left(G_{M}(t)+G_{E}(t)\right),
\end{gathered}
$$

where $t=\left(E_{\Delta}-E_{N}\right)^{2}-\mathbf{Q}^{2}$ and

$$
J^{+}(0)=\frac{J^{1}(0)+i J^{2}(0)}{\sqrt{2}}, \quad A=\frac{E_{\Delta}+m_{N}}{2 m_{N}} \sqrt{2 E_{\Delta}\left(Q^{0}-m_{N}\right)} .
$$

In the following, we denote them as $F_{i}, \mathrm{i}=1,2,3$.

In order to extract these matrix elements on the lattice, we define the following local field operators,

$$
\begin{aligned}
O_{3 / 2}(X)= & \frac{1}{2}\left(1+\Sigma_{3}\right) \frac{1}{2}\left(1+\gamma_{4}\right) \frac{1}{\sqrt{2}}\left(O^{1}(X)-i \Sigma_{3} O^{2}(X)\right), \\
O_{1 / 2}(X)= & \frac{1}{2}\left(1-\Sigma_{3}\right) \frac{1}{2}\left(1+\gamma_{4}\right) \frac{1}{\sqrt{2}}\left(O^{1}(X)+i \Sigma_{3} O^{2}(X)\right), \\
& \tilde{O}_{1 / 2}(X)=\frac{1}{2}\left(1+\Sigma_{3}\right) \frac{1}{2}\left(1+\gamma_{4}\right) O^{3}(X), \\
\bar{\psi}_{ \pm 1 / 2}(Y)= & \bar{\psi}(Y) \frac{1}{2}\left(1 \pm \Sigma_{3}\right) \frac{1}{2}\left(1+\gamma_{4}\right), \quad \Sigma_{3}=\operatorname{diag}\left(\sigma_{3}, \sigma_{3}\right),
\end{aligned}
$$

which produce the $\Delta$ and nucleon particles with a given spin projection, denoted by the lower index on the 1.h.s of Eq. (6). We refer the reader to Ref. [6] for more details

The quantities $F_{i}$ can be measured on the lattice, however, one gets the matrix elements of the current between a given eigenstate of the finite-volume Hamiltonian and a one-nucleon state. The dependence of the energy $E_{\Delta}$ on the volume is not suppressed exponentially (unlike the case of a stable $\Delta$ ), but only as inverse power of the volume [14]. The same statement holds for the matrix elements $F_{i}$.

\section{Multipoles and form factors}

The volume dependence of the quantities $F_{i}$ arises, due to the initial-state pion-nucleon interaction. The essence of the Lellouch-Lüscher method is to exactly cancel this dependence on the volume. 


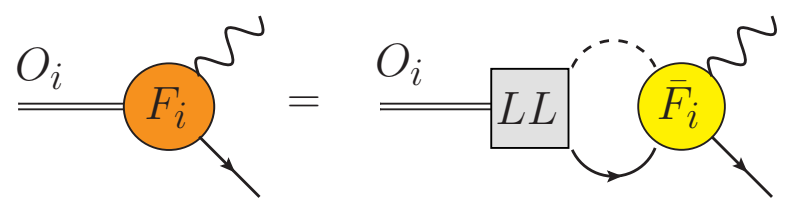

Figure 1. The $\Delta N \gamma^{*}$ vertex in a finite volume

The diagrammatic representation of the $\Delta N \gamma^{*}$ vertex within the non-relativistic effective field theory in a finite volume ${ }^{1}$ is shown in Fig. 1. The grey box contains all two-particle reducible diagrams, which are summed up to the well-known Lellouch-Lüscher factor. This factor contains all dependence on the volume. The amplitudes $\bar{F}_{i}$ are volume-independent (up to the contributions exponentially suppressed in volume). Moreover, they can be related to particular $\gamma^{*} N \rightarrow \pi N$ helicity amplitudes, to which the $\Delta$ resonance might contribute (see Ref. [6]).

Finally, we summarize our results on the $\Delta N \gamma^{*}$ transition form factors:

1. The matrix elements $F_{i}=F_{i}(p,|\mathbf{Q}|)$ are functions of the relative momenta in the $\pi N$ system $p$ and $|\mathbf{Q}|$. In order to extract the form factors at the resonance pole (see below), the quantities $F_{i}$ must be measured at different values of $p$ in the $\Delta$-region, keeping $|\mathbf{Q}|$ fixed. This can be achieved, e.g., by applying asymmetric boxes or the twisting in the nucleon line. The scattering phase shift $\delta(p)$ should be calculated through the Lüscher equation at the same values of $p: \delta(p)+\phi(q)=n \pi, n=0,1, \ldots$, where $q=p L / 2 \pi$ and $\phi(q)$ is related to the Lüscher zeta-function.

2. The helicity amplitudes $\mathcal{A}_{i}$ for pion photo(electro)production in the elastic region at a given discrete momenta $p$ are given by

$$
\mathcal{A}_{i}(p,|\mathbf{Q}|)=e^{i \delta(p)} V^{1 / 2}\left(\frac{p^{2}}{2 \pi|d \delta(p) / d p+d \phi(q) / d p|}\right)^{-1 / 2}\left|F_{i}(p,|\mathbf{Q}|)\right|,
$$

where $V$ is a volume. The relation between the helicity and multipole amplitudes can be found in Refs. [6,9].

3. The $\Delta N \gamma^{*}$ matrix elements $F_{i}^{A}\left(p_{A},|\mathbf{Q}|\right)$, defined at real energies, are given by Eq. (3), with additional index $i=1,2,3$ attached.

4. The extraction of the $\Delta N \gamma^{*}$ matrix elements at the resonance pole proceeds as follows. One first calculates the irreducible amplitudes $\bar{F}_{i}(p,|\mathbf{Q}|)$ from the equation

$$
\bar{F}_{i}(p,|\mathbf{Q}|)=V^{1 / 2}\left(\frac{\cos ^{2} \delta(p)}{|d \delta(p) / d p+d \phi(q) / d p|} \frac{p^{2}}{2 \pi}\right)^{-1 / 2} F_{i}(p,|\mathbf{Q}|) .
$$

5. Since the functions $p^{3} \cot \delta(p) \bar{F}_{i}(p,|\mathbf{Q}|)$ are low-energy polynomials in $p^{2}$ (see Ref. [6]), they can be fitted by the effective-range expansion to lattice data:

$$
p^{3} \cot \delta(p) \bar{F}_{i}(p,|\mathbf{Q}|)=A_{i}(|\mathbf{Q}|)+p^{2} B_{i}(|\mathbf{Q}|)+\cdots .
$$

6. The resonance matrix elements at the pole are evaluated by substitution

$$
F_{i}^{R}\left(p_{R},|\mathbf{Q}|\right)=i p_{R}^{-3} Z_{R}^{1 / 2}\left(A_{i}(|\mathbf{Q}|)+p_{R}^{2} B_{i}(|\mathbf{Q}|)+\cdots\right),
$$

\footnotetext{
${ }^{1}$ For alternative, but equivalent approaches, see, e.g., Refs. [4, 15, 16].
} 


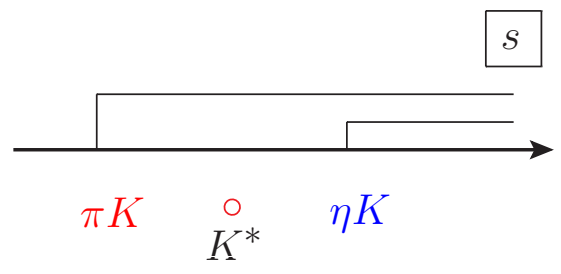

Figure 2. Thresholds near $K^{*}(892)$ resonance

where $p_{R}$ is the $\Delta$ resonance complex pole position, and $Z_{R}$ is given by

$$
Z_{R}=\left.\left(\frac{p}{8 \pi E}\right)^{2}\left(\frac{16 \pi p^{3} E^{3}}{w_{N} w_{\pi}\left(2 p d\left(p^{3} \cot \delta(p)\right) / d p+3 i p^{2}\right)}\right)\right|_{p=p_{R}} .
$$

Here, the quantities $w_{N}, w_{\pi}$ denote the on-shell energies of the nucleon and pion, respectively. The pole position $p_{R}$ must be found separately, e.g., by applying the effective-range expansion:

$$
-\frac{1}{a}+\frac{1}{2} r p_{R}^{2}+\cdots=-i p_{R}^{3}
$$

where the constants $a, r$ should be fitted.

7. Finally, the form factors can be derived from Eq. (4).

\section{Summary and Outlook}

This write-up summarizes our findings on the theoretical framework for the extraction of the $\Delta N \gamma^{*}$ transition form factors on the lattice. The framework is based on the non-relativistic effective field theory in a finite volume. We have derived the analogue of the Lellouch-Lüscher formula for the photo(electro)production amplitude in the elastic region. The prescription for the extraction of the form factors at the resonance pole has been also given.

The result might be improved in one particular way. It would be important to consider the $\Delta$ resonance in the moving frames. Then, one can carry out a similar procedure as given above.

The rare $B \rightarrow K^{*}(892) l^{+} l^{-}$decay is another example of the phenomenologically interesting process. It can be studied along the same lines. However, this problem is more complicated, due to the presence of the second $K \eta$ channel (see Fig. 2). The multichannel generalization of the LellouchLüscher method has been derived in Refs. [17, 18]. On the contrary, the extraction of the form factors at the resonance pole in the multichannel case has not been studied before. Recently, we have filled this gap and considered the $\pi K-\eta K$ coupled-channel system in a finite volume [19]. A similar theoretical framework might be useful for the extraction of the electromagnetic form factors of the $\Lambda(1405)$ resonance on the lattice [20].

\section{Acknowledgments}

We acknowledge the support by the DFG (CRC 16, "Subnuclear Structure of Matter") and by the Bonn-Cologne Graduate School of Physics and Astronomy. This research is supported in part by Volkswagenstiftung under contract no. 86260. 


\section{References}

[1] C. Alexandrou, G. Koutsou, J. W. Negele, Y. Proestos and A. Tsapalis, Phys. Rev. D 83 (2011) 014501

[2] C. Alexandrou, G. Koutsou, H. Neff, J. W. Negele, W. Schroers and A. Tsapalis, Phys. Rev. D 77 (2008) 085012

[3] C. E. Carlson and M. Vanderhaeghen, Phys. Rev. Lett. 100 (2008) 032004

[4] L. Lellouch and M. Lüscher, Commun. Math. Phys. 219 (2001) 31

[5] V. Bernard, D. Hoja, U.-G. Meißner and A. Rusetsky, JHEP 1209 (2012) 023

[6] A. Agadjanov, V. Bernard, U.-G. Meißner and A. Rusetsky, Nucl. Phys. B 886 (2014) 1199

[7] M. T. Hansen and S. R. Sharpe, Phys. Rev. D 86 (2012) 016007.

[8] R. A. Briceno, M. T. Hansen and A. Walker-Loud, arXiv:1406.5965 [hep-lat] (2014).

[9] I. G. Aznauryan, V. D. Burkert and T. -S. H. Lee, arXiv:0810.0997 [nucl-th].

[10] D. Drechsel, O. Hanstein, S. S. Kamalov and L. Tiator, Nucl. Phys. A 645 (1999) 145

[11] R. L. Workman, L. Tiator and A. Sarantsev, Phys. Rev. C 87 (2013) 6, 068201

[12] G. Colangelo, J. Gasser, B. Kubis and A. Rusetsky, Phys. Lett. B 638 (2006) 187

[13] J. Gasser, B. Kubis and A. Rusetsky, Nucl. Phys. B 850 (2011) 96

[14] M. Lüscher, Nucl. Phys. B 354 (1991) 531

[15] C. H. Kim, C. T. Sachrajda and S. R. Sharpe, Nucl. Phys. B 727 (2005) 218

[16] N. H. Christ, C. Kim and T. Yamazaki, Phys. Rev. D 72 (2005) 114506

[17] M. T. Hansen and S. R. Sharpe, Phys. Rev. D 86 (2012) 016007

[18] R. A. Briceño, M. T. Hansen and A. Walker-Loud, Phys. Rev. D 91 (2015) 3, 034501

[19] A. Agadjanov, V. Bernard, U.-G. Meißner and A. Rusetsky, in preparation.

[20] B. J. Menadue, W. Kamleh, D. B. Leinweber, M. Selim Mahbub and B. J. Owen, PoS LATTICE 2013 (2014) 280 